
2019, Vol. 6, No. 1, 56-67.

DOI: https://doi.org/10.17979/reipe.2019.6.1.4910

\title{
Tradução e adaptação para português da Vanderbilt ADHD Diagnostic Teacher Rating Scale: um estudo piloto
}

\section{Translation and adaptation to Portuguese of Vanderbilt ADHD Diagnostic Teacher Rating Scale: A pilot study}

\author{
Luís Oliveira (D)*, Marcelino Pereira (D)**, Teresa Medeiros (D)***, Ana Serrano (D)* \\ *Universidade do Minho, **Universidade de Coimbra, ***Universidade dos Açores
}

\begin{abstract}
Resumo
Este estudo integra uma investigação mais ampla, cujo propósito foi analisar o impacto da Perturbação de Hiperatividade/Défice de Atenção (PHDA) em contexto escolar, a nível académico, comportamental e social. A Vanderbilt Adhd Diagnostic Teacher Rating Scale (VADTRS) - Escala Vanderbilt de Diagnóstico de PHDA para Professores (EVDPP) - foi traduzida e adaptada para Português Europeu. 76 professores do $1 .^{\circ}$ ciclo da ilha de São Miguel (Açores), preencheram a EVDPP para 105 alunos diagnosticados com PHDA ou em avaliação, com idades compreendidas entre os 6 e os 11 anos, $85.7 \%$ são do género masculino e $14.3 \%$ do feminino. Os resultados demonstram a validade interna da escala, com alfas de Chronbach a situarem-se entre os .80 e .94 nas diferentes dimensões, corroborando outros estudos internacionais. Estes dados preliminares sugerem que a EVDPP pode ser um instrumento extremamente útil para as equipas multidisciplinares na sinalização, diagnóstico e caraterização dos principais problemas da PHDA em contexto escolar.
\end{abstract}

Palavras chave: Ensino Básico, crianças, avaliação, PHDA

Abstract

This study integrates a broader investigation, whose purpose was to analyze the impact of Attention Deficit Hyperactivity Disorder (ADHD) within a school context, at an academic, behavioral and social level. The Vanderbilt ADHD Diagnostic Teacher Rating Scale (VADTRS) has been translated and adapted into Portuguese European, using cross-cultural translation method of back-translation and decentering, proposed by Brislin $(1970,1976)$. A total of 76 teachers from the 1st cycle of the island of São Miguel (Azores) filled the EVDPP for $105(\mathrm{n}=105)$ students diagnosed (or under evaluation) for diagnosis of ADHD, aged between 6

Luís Oliveira (DD) e Ana Serrano (D): Instituto de Educação (IE), Universidade do Minho Campus de Gualtar. 4710-057 Braga, Portugal. Marcelino Pereira (D): Faculdade de Psicologia e Ciências da Educação, Universidade de Coimbra. R. Colégio Novo, 3000-802 Coimbra, Portugal.

Teresa Medeiros (D): Departamento de Ciências Sociais e Humanas, Universidade dos Açores, Campus de Ponta Delgada. 9501-801 Ponta Delgada, Portugal.

Correspondência relativa a este artigo: Luís Oliveira - luismicaelense@hotmail.com 
and 11 years old, $85.7 \%$ are male gender and $14.3 \%$ are female. The statistical method used to evaluate the internal consistency of the instrument was the Chronbach's alpha. The results demonstrate the internal validity of the scale, with Chronbach's alphas being between .80 and .94 in the different dimensions, corroborating other international studies. These preliminary data suggest that EVDPP can be an extremely useful tool for the multidisciplinary teams of Portuguese schools (in particular psychologists), in the signaling, diagnosis and characterization of the main problems of the PHDA in this context.

Keywords: Basic Education; children; assessment; ADHD

Por definição, a Perturbação de Hiperatividade/Défice de Atenção (PHDA) significa "[...] um atraso evolutivo que afeta a capacidade da criança para regular o comportamento, controlar o nível de atividade, inibir reações impulsivas ou manter a atenção" (Barkley e Edwards, 2006/2008, p. 375). Estima-se que afete cerca de 5\% das crianças (APA, 2014) e conhece-se o risco elevado da existência de outras comorbilidades de natureza internalizante e externalizante (e.g., perturbação de ansiedade - PA; perturbação depressiva - PD; perturbação desafiante de oposição - PDO; perturbação do comportamento - PC, perturbação da aprendizagem específica PAE), assim como outros problemas associados (e.g., académicos, comportamentais e sociais), que agravam o prognóstico e que poderão manter-se na idade adulta (Faraone et al., 2015). "O ambiente escolar pode ser um lugar difícil para as crianças com PHDA porque os sintomas são diametralmente opostos às expetativas de uma sala de aula tradicional (...)" (Shillingford-Butler e Theodore, 2013, p. 236). Do ponto de vista teórico ou concetual seria difícil projetar um ambiente mais problemático para os alunos com PHDA que, a típica sala de aula, onde são obrigados a estar quietos, ouvir atentamente as instruções académicas, completar autonomamente as tarefas escolares, esperar pela sua vez, relacionar-se adequadamente com os colegas e professores, etc. Tais requisitos são extraordinariamente difíceis de cumprir, especialmente devido aos problemas que as pessoas com PHDA apresentam no funcionamento executivo (DuPaul et al., 2014a). Estas dificuldades não só prejudicam o desempenho dos alunos com esta perturbação, como constituem um enorme desafio para os psicólogos e outros agentes escolares que trabalham com esta população (DuPaul e Jimerson, 2014).

Os professores desempenham um papel fundamental na sinalização e identificação dos alunos com PHDA, sendo a sua participação normalmente exercida através do preenchimento de escalas e questionários devidamente aferidos e adaptados para a população a que se destinam (DuPaul e Jimerson, 2014; DuPaul, Reid, Anastopoulos, e Power, 2014b; Salmon e Kirby, 2009).

Ao longo de décadas de investigação desenvolveram-se novos instrumentos de avaliação comportamental da PHDA (inventários e escalas) que fornecem, de forma sistematizada, informação valiosa aos profissionais que se dedicam à avaliação da PHDA, nomeadamente os pediatras, psicólogos, psiquiatras e outras especialidades médicas, numa lógica multidisciplinar, uma vez que o diagnóstico final resulta da confluência de diferentes práticas clínicas devidamente estabelecidas, incluindo o preenchimento de escalas de avaliação por parte dos pais e professores (Oliveira et al., 2016). Não existe consenso absoluto sobre quais os mais indicados, pois estes diferem, quer na amplitude dos comportamentos abordados, quer suas caraterísticas psicométricas. Entre os instrumentos mais conhecidos estão as escalas desenvolvidas por Keith Conners (e.g., Conners, 1969), da qual derivam outras versões abreviadas mais recentes e fáceis de aplicar. Uma destas, a versão para professores da Escala de Conners - Versões Revistas: Forma Reduzida - Conners Rating Scale-Revised: Short Form, datada de 1997, foi aferida para a população portuguesa (Rodrigues, 2007). Apesar da sua ampla utilização, as escalas de Conners não se focam nos sintomas específicos enumerados no DSM (i.e., não utilizam, em rigor, os critérios de diagnóstico do DSM, especificamente do DSM-5), nem incluem, de forma abrangente, a descrição de outros comportamentos/problemas geralmente associados à PHDA (e.g., ansiedade, depressão, desempenho académico, 
entre outros). Neste contexto, foram concertados esforços no sentido de se obter um instrumento robusto (validado empiricamente) e que correspondesse de forma alargada aos objetivos deste estudo.

No seguimento da pesquisa efetuada, a escala que motivou maior consenso entre os autores desta investigação foi a Vanderbilt ADHD Diagnostic Teacher Rating Scale (VADTRS) (Wolraich et al., 1998) - Escala Vanderbilt de Diagnóstico de PHDA para Professores, adiante designada EVDPP. Esta escala contém a informação necessária para um diagnóstico da PHDA baseado nos critérios do DSM-5 e para a discriminação de comorbilidades que lhe estão frequentemente associadas (Wolraich et al., 1998). Apesar da escala não estar aferida para a população portuguesa existem estudos internacionais que evidenviam a robustez clínica do instrumento para cumprir os propósitos anteriormente descritos (e.g., Wolraich et al., 2003a).

O presente estudo tem como objetivos a tradução e adaptação da EVDPP para Português Europeu.

\section{Método}

\section{Participantes}

Participaram neste estudo105 alunos $(\mathrm{n}=105)$ do $1 .^{\circ}$ ciclo do ensino básico, com idades entre os 6 e os 11 anos $\left(M=8.59 ; D P=1.30 ; n_{6 \mathrm{a}}=4 ; n_{7 \mathrm{a}}=21 ; n_{8 \mathrm{a}}=24 ; n_{9 \mathrm{a}}=29 ; n_{10 \mathrm{a}}=19 ; n_{11 \mathrm{a}}=8\right)$, dos quais $15(14.3 \%)$ são do género feminino e $90(85.7 \%)$ do género masculino, resultados que entrocam nas evidências que demonstram que a PHDA é mais frequente nos rapazes do que nas raparigas (Erskine et al., 2013; Willcutt, 2012). A distribuição da frequência escolar mostra que 24 alunos $\left(n_{1 .}{ }^{\circ}=24\right)$ estavam a frequentar o $1 .^{\circ}$ ano de escolaridade, correspondendo assim a $22.9 \%$ do total de participantes, ao passo que $38\left(n_{2 .}{ }^{\circ}=38\right)$ se encontravam no $2 .^{\circ}$ ano $(36.2 \%), 21\left(n_{3 .}{ }^{\circ}=\right.$ 21) estavam no $3 .^{\circ}$ ano $(20 \%)$, e $22\left(n_{4} .^{\circ}=22\right)$ frequentavam o $4 .^{\circ}$ ano, o equivalente a $20.9 \%$.

Os referidos alunos estavam distribuídos por 42 escolas públicas/privadas da ilha de São Miguel e foram avaliados através da EVDPP por 76 professores, titulares de turma dos alunos visados.

\section{Instrumento (tradução e adaptação)}

À semelhança das escalas de Conners, existem duas versões das escalas Vanderbilt, para pais (Wolraich et al., 2003b) e para professores (Wolraich et al., 1998).

Para além dos 18 sintomas típicos da PHDA reproduzidos no DSM-5, a EVDPP contempla ainda um grupo de dez itens referentes a síntomas de Perturbação Desafiante de Oposição e Perturbação do Comportamento (PDO/PC) e outros sete itens de Perturbação de Ansiedade e Perturbação Depressiva (PA/PD), que são avaliados pelos professores através de uma escala de Lickert pontuada de 0 a 3, sendo que o zero revela a inexistência de síntomas e o três demonstra a sua presença muito frequente. Adicionalmente, incorpora duas subescalas que analisam o desempenho da criança na escola: três itens que dizem respeito a problemas na aprendizagem (PA) - leitura, matemática e expressão escrita - e cinco itens que classificam os problemas comportamentais (PC) em sala de aula - competências sociais de relacionamento com os pares, capacidade de organização e conclusão das tarefas, cumprimento de ordens/regras e perturbação das aulas. Estes oito itens são avaliados através de uma escala de Lickert de cinco pontos (de 1 a 5), que retrata se o desempenho é problemático (pontuações de 1 e 2), se está na média (pontuações de 3) ou acima da média (pontuações de 4 e 5).

Os resultados de diferentes estudos mostram que a EVDPP reúne excelentes qualidades psicométricas. Os seus autores reportam uma boa consistência interna, com os alfas de Cronbach a variarem entre .80 e .95 para as diferentes dimensões (Wolraich et al., 1998). Outras investigações apontam coeficientes alfas de Cronbach que oscilam entre .71 e .96 (Wolraich et al., 2003a; Wolraich, Bard, Neas, Doffing, e Beck, 2013).

Por outro lado, a investigação também aponta para uma boa validade (discriminativa, convergente e preditiva), bem como sensibilidade e especificidade satisfatórias. Para a validade do construto, um modelo de quatro fatores (i.e., desatenção; hiperatividade/impulsividade; problemas de desafio/oposição; e problemas de 
ansiedade/depressão) configurou-se ajustado ao instrumento (Wolraich et al., 2013).

Especificamente para os sintomas da PHDA foram testados três modelos diferentes (i.e., 18 sintomas da PHDA; desatenção e hiperatividade/impulsividade; e desatenção, hiperatividade, impulsividade), sendo que o modelo de dois fatores (i.e., desatenção e hiperatividade/impulsividade) foi o que obteve melhor desempenho, explicando 68 a 77\% da variância (Wolraich et al., 2003a).

A validade convergente, tendo como referência o "Questionário de Capacidades e Dificuldades" (Strengths and Difficulties Questionnaire) para os quatro fatores foi elevada $(r>.72)$. Em relação à validade preditiva, obteve-se uma sensibilidade de .69 e especificidade de .84 , valor preditivo positivo de .32 e valor preditivo negativo de .96 , respetivamente, ao antever/excluir casos de PHDA entre crianças cujos pais completaram uma entrevista de diagnóstico estruturada (Diagnostic Interview Schedule for Children-IV) (Wolraich et al., 2013).

Por sua vez, os itens de desempenho da leitura e expressão escrita apresentaram-se como excelentes preditores em relação à presença de Perturbações de Aprendizagem Específicas (i.e., na leitura e escrita), quando acompanhados com uma entrevista acerca do desempenho escolar do aluno e uma revisão ao seu histórico escolar (Langberg, Vaughn, Brinkman, Froehlich, e Epstein, 2010). Com efeito, um ponto de corte de 4 para os itens de leitura e escrita apresentou um ótimo poder preditivo negativo (0.80) no afastamento de uma comorbilidade de PAE da leitura, assim como um excelente poder preditivo negativo (1.00) para uma PAE da escrita (Langberg et al., 2010).

Feita a apresentação do instrumento, procedemos, em seguida, à descrição da sua tradução e adaptação. Depois de obtida a autorização ao seu autor para a sua utilização nesta investigação, a EVDPP foi traduzida para português e alvo de ligeiras adaptações, de acordo com os métodos de tradução intercultural de instrumentos, retrotradução e descentramento, propostos por Brislin $(1970,1976)$.

Numa fase inicial o instrumento foi traduzido por um tradutor bilingue acreditado (freelancer), residente na ilha de São Miguel, e revisto por um tradutor/revisor pertencente à bolsa de tradutores de uma empresa especializada em tradução. O processo de tradução/revisão visou comparar/corrigir eventuais imprecisões. As duas versões foram consideradas equivalentes (i.e., lexical e gramaticalmente) e, portanto, satisfatórias. Não obstante, como é referido por Brislin (1970), a tradução bilingue pode manter muitas das formas gramaticais do documento de origem (facilitando desta forma a tarefa de retrotradução), mas não cumprir, de modo fidedigno, o propósito de questionar os monolingues da língua alvo (i.e., quando uma língua contém especificidades que não podem ser traduzidas para outro idioma sem alguma modificação).

Na medida em que o investigador principal tem um domínio aceitável da língua inglesa, está familiarizado com os termos técnicos e conhece a lista de sintomas do DSM-5 utilizada no instrumento original (versão do DSM-5 existente em português), predispôs-se simultaneamente à tarefa da sua tradução.

Posteriormente, as duas versões traduzidas acima descritas, foram analisadas por um especialista do ramo (Dr. José Boavida Fernandes, médico pediatra e responsável da unidade de Hiperatividade do Centro de Desenvolvimento da Criança Dr. Luís Borges, do Hospital Pediátrico de Coimbra). A sua vasta experiência clínica neste domínio torna-o profundo conhecedor do conteúdo e semântica dos itens presentes neste tipo de escalas em diferentes línguas, particularmente no português e inglês, qualidades necessárias para a tarefa seguinte da validação/adequação dos itens. Este processo foi essencial para perceber quais os termos das duas traduções quando comparados com o texto original - estavam mais equivalentes entre si, no sentido de terem, efetivamente, o mesmo significado referencial e serem, simultaneamente, culturalmente relevantes e apropriados. A comparação das duas traduções resultou numa versão de consenso, que se considerou ser a que transparecia de forma mais profícua as intenções de avaliação do documento original. No Anexo I (cf. Apêndices) é possível verificar o resultado final da tradução efetuada por comparação à original.

No que respeita à adequação dos itens, ligeiras adaptações foram efetuadas, principalmente nos itens formulados 
na negativa, uma vez que podem dificultar a interpretação e consequente decisão da resposta, pelo que relativamente a estes itens adotou-se uma sintaxe específica, convertendo-os em afirmações positivas de modo a evitar o potenciar de falsos negativos.

Esta última versão do instrumento, por sua vez, foi alvo de uma retrotradução, efetuada por um tradutor bilingue de língua materna inglesa (americano), qualificado e com vasta experiência em tradução, a residir nos EUA. A análise comparativa das duas versões, que se encontra no Anexo II (cf. Apêndices), não regista diferenças substanciais entre a versão original e a retrotradução.

As pequenas divergências verificadas não colocaram em causa o significado dos itens e derivaram do método de "descentramento".

O processo culminou com o envio da versão final da tradução (portuguesa), assim como a sua retrotradução (inglês - americano), ao autor da escala, Professor Mark Wolraich, que facultou ao investigador principal desta investigação um parecer favorável.

\section{Procedimentos}

O investigador principal, depois de garantida as autorizações da Diretora Regional de Educação dos Açores, dos presidentes dos conselhos executivos dos agrupamentos das escolas públicas da ilha de São Miguel, assim como aos diretores das escolas privadas, dirigiu-se presencialmente a cada uma das escolas e articulou com o coordenador uma data e hora para a realização de uma reunião. Aí questionou todos professores do $1 .{ }^{\circ}$ Ciclo se tinham alunos diagnosticados com PHDA ou em fase de avaliação. Todos aqueles que responderam afirmativamente foram convidados a completar a EVDPP.

Explicado o objetivo do seu preenchimento, foram igualmente instruídos para a realização desta tarefa. Foilhes solicitado que devolvessem o questionário sem a identificação da criança, em cumprimento com as questões éticas da investigação, em particular de acordo com as normas da Subcomissão de Ética para as Ciências Sociais e Humanas (SECDH) da Universidade do Minho.

As escalas foram distribuídas faseadamente, tendo ficado acordado entre o investigador e os professores, que a sua recolha seria feita uma semana após a entrega. O período de recolha da totalidade das escalas situou-se entre janeiro e julho de 2015. Optou-se por iniciar este processo no segundo semestre do ano escolar, período em que os professores já detinham informação relevante quanto ao comportamento/desempenho dos seus alunos.

Os dados que a seguir apresentamos foram obtidos com o recurso ao IBM -Statistical Package for the Social Sciences (SPSS) - Versão 21. O teste estatístico utilizado para analisar a consistência interna da EVDPP foi o alfa de Cronbach (cf. Tabela 1).

Para a interpretação dos coeficientes, assumimos a definição de Marôco (2014, p.23, 24) que nos diz que "De uma forma geral nas ciências sociais e humanas, mas sem definições precisas, consideramos que as correlações são: (i) fracas quando o valor absoluto de $\mathrm{r}(|\mathrm{r}|)$ é inferior a 0.25 ; (ii) são moderadas para $0.25 \leq|\mathrm{r}| \leq 0.5$; (iii) são fortes para $0.5 \leq|\mathrm{r}| \leq 0.75$ e (iv) são muito fortes se $|\mathrm{r}| \geq 0.75$."

\section{Resultados}

\section{Consistência Iinterna}

Os resultados deste estudo confirmam a boa consistência interna da EVDPP, com alfas de Cronbach a variarem entre .80 e .93 nas diferentes dimensões - sintomas de desatenção (.84); sintomas de hiperatividade/impulsividade (.91); sintomas de PDO/PC (.93); sintomas de PA/PD (.87); problemas na aprendizagem - PA (.84) e problemas comportamentais - PC (.80).

As principais componentes de análise para a medida da consistência interna (coeficiente alfa) são apresentadas na Tabela 1. 
Tabela 1

Correlações entre os itens e total corrigido, coeficientes alfa de Cronbach excluindo o item e nota global do alfa de Cronbach para cada dimensão $(N=105)$

\begin{tabular}{|c|c|c|}
\hline Itens & $\begin{array}{l}\text { Correlação Item- } \\
\text { Total corrigido }\end{array}$ & $\begin{array}{l}\text { Alfa de Cronbach } \\
\text { excluindo o item }\end{array}$ \\
\hline \multicolumn{3}{|l|}{ Dimensão sintomas Desatenção $(\alpha$ global $=.84)$} \\
\hline $\begin{array}{l}\text { 1. Tem dificuldade em prestar atenção aos detalhes ou comete erros por descuido nos } \\
\text { trabalhos escolares }\end{array}$ & .55 & .83 \\
\hline 2. Tem dificuldade em manter a atenção em tarefas ou atividades & .63 & .82 \\
\hline 3. Parece não ouvir quando se lhe fala diretamente & .46 & .84 \\
\hline $\begin{array}{l}\text { 4. Tem dificuldade em seguir as instruções e em terminar os trabalhos escolares (não por que } \\
\text { se recuse ou por não compreender) }\end{array}$ & .62 & .82 \\
\hline 5. Tem dificuldade em organizar tarefas e atividades & .61 & .82 \\
\hline $\begin{array}{l}\text { 6. Evita, não gosta ou mostra-se relutante em envolver-se em tarefas que exijam um esforço } \\
\text { mental sustentado }\end{array}$ & .51 & .83 \\
\hline $\begin{array}{l}\text { 7. Perde material necessário à realização de tarefas ou atividades (trabalhos da escola, lápis } \\
\text { ou livros) }\end{array}$ & .57 & .83 \\
\hline 8. Distrai-se facilmente com estímulos externos & 60 & .83 \\
\hline 9. É esquecido/a nas atividades quotidianas & .59 & .83 \\
\hline \multicolumn{3}{|l|}{ Dimensão sintomas Hiperatividade /Impulsividade $(\alpha$ global $=.91)$} \\
\hline 10. Mexe constantemente as mãos ou pés ou não pára quieto/a na cadeira & .61 & .91 \\
\hline 11. Levanta-se na sala de aula ou noutras situações em que devia permanecer sentado/a & 69 & .91 \\
\hline 12. Corre ou trepa excessivamente em situações em que devia permanecer sentado/a & 67 & .91 \\
\hline 13. Tem dificuldade em jogar ou em envolver-se tranquilamente em atividades de lazer & .61 & .91 \\
\hline $\begin{array}{l}\text { 14. Está sempre em movimento ou age frequentemente como se fosse "movido/a por um } \\
\text { motor" }\end{array}$ & .79 & .90 \\
\hline 15. Fala excessivamente & 68 & .91 \\
\hline 16. Responde precipitadamente antes de ouvir as questões até ao fim & .66 & .91 \\
\hline 17. Tem dificuldade em esperar pela sua vez & .82 & .90 \\
\hline 18. Interrompe os outros ou intromete-se (por exemplo, em conversas ou jogos) & .75 & .90 \\
\hline \multicolumn{3}{|l|}{ Dimensão sintomas Perturbação de Oposição/Desafio e Perturbação Comportamental $(\alpha$ global = .93) } \\
\hline 19. Perde o controlo & .65 & .93 \\
\hline 20. Desafia abertamente ou recusa-se a cumprir as ordens ou regras dos adultos & .78 & .92 \\
\hline 21. Está zangado/a ou ressentido/a & .73 & .93 \\
\hline 22. É rancoroso/a e vingativo/a & .81 & .92 \\
\hline 23. Assusta, ameaça ou intimida os outros & .88 & .92 \\
\hline 24. Inicia lutas físicas & .81 & .92 \\
\hline 25. Mente para conseguir bens ou favores ou para evitar obrigações (isto é, engana os outros) & 69 & .93 \\
\hline 26. É fisicamente cruel para as pessoas & .80 & .92 \\
\hline 27. Rouba objetos de valor significativo & 60 & .93 \\
\hline 28. Destrói deliberadamente os bens dos outros & .70 & .93 \\
\hline \multicolumn{3}{|l|}{ Dimensão síntomas Perturbação de ansiedade e Perturbação Depressiva $(\alpha$ global $=.87)$} \\
\hline 29. É temeroso/a, ansioso/a ou preocupado/a & .50 & .87 \\
\hline 30. É tímido/a ou fica envergonhado/a facilmente & .59 & .86 \\
\hline 31. Tem medo de experimentar coisas novas por receio de cometer erros & .74 & .84 \\
\hline 32. Sente-se inútil ou inferior & .76 & .84 \\
\hline 33. Culpabiliza-se pelos problemas, sente-se culpado/a & .58 & .86 \\
\hline 34. Sente-se sozinho/a, indesejado/a ou pouco amado/a; reclama que "ninguém gosta dele/a" & .65 & .85 \\
\hline 35. Está triste, infeliz ou deprimido/a & .72 & .84 \\
\hline \multicolumn{3}{|l|}{ Dimensão Problemas na aprendizagem $(\alpha$ global $=.84)$} \\
\hline 1. Leitura & .75 & .73 \\
\hline 2. Matemática & .61 & .86 \\
\hline 3. Expressão Escrita & .78 & .72 \\
\hline \multicolumn{3}{|l|}{ Dimensão problemas comportamentais $\alpha$ global $=.80$ ) } \\
\hline 1. Relacionamento com os colegas & .69 & .72 \\
\hline 2. Cumprimento de ordens/regras & .72 & .71 \\
\hline 3. Perturbação das aulas & .61 & .75 \\
\hline 4. Conclusão de tarefas & .42 & .80 \\
\hline 5. Capacidade de organização & .47 & .79 \\
\hline
\end{tabular}


Como é possível verificar na Tabela 1, apenas três itens apresentam correlações com o total corrigido abaixo de .50, sendo que os restantes correlacionam-se com o total corrigido acima deste valor, pelo que, no presente caso, podemos constatar que a correlações entre os itens das diferentes dimensões e o total corrigido são elevadas, o que nos permite aferir a validade interna destes mesmos itens. Por sua vez, os coeficientes de Cronbach excluindo cada um dos itens não aumentam, o que evidencia a importância e o seu contributo para a consistência interna da EVDPP. O alfa de Cronbach global mais baixo foi .80, que é considerado muito forte.

\section{Discussão}

O interesse da EVDPP não se confina a trabalhos de investigação. A confirmação de uma boa validade convergente e confiabilidade aceitável do constructo fazem deste instrumento uma ferramenta útil para o diagnóstico da PHDA (Wolraich et al., 2013). Os resultados mostram simultaneamente que os itens de desempenho da leitura e escrita podem ser utilizados pelos médicos para determinar que crianças não necessitam de ser referenciadas para uma avaliação deste tipo de comorbilidade (i.e., PAE da leitura e escrita). Dada a limitação das escolas e dos psicólogos em completar testes psicoeducacionais esta informação clínica ganha especial importância para a identificação das crianças que efetivamente necessitam de uma avaliação mais rigorosa em relação à presença de PAE (Langberg et al., 2010). As opções metodológicas utilizadas para a tradução e adaptação da EVDPP para português revelaram-se adequadas, tendo em conta a robustez dos indicadores obtidos relativos à consistência interna do instrumento, por comparação a outros estudos internacionais que analisaram esta componente, assim como pela fácil leitura e interpretação dos itens por parte dos professores que avaliaram os participantes deste estudo.

Os resultados obtidos parecem desta forma sugerir as boas qualidades psicométricas da EVDPP, todavía, outras etapas deverão ser asseguradas em futuras investigações, como a análise da sensibilidade ou da validade convergente, de forma a compreender proficuamente o comportamento do instrumento na população portuguesa, com vista à sua normalização. Cientes das limitações inerentes a um estudo desta natureza, importa, porém, salientar que a EVDPP pode assumir-se como um instrumento bastante útil para alguns profissionais das escolas portuguesas, tanto na sinalização dos problemas subjacentes à problemática da PHDA em contexto escolar, como consequentemente no planeamento da prática pedagógica que os professores deverão implementar com estas crianças. Com efeito, o acesso aos elementos de diagnóstico mais recomendados não é da exclusividade dos psicólogos, mas é reconhecido que estes, dada a sua formação académica, podem prestar um excelente contributo, uma vez que parte da sua preparação foi direcionada para o uso de técnicas e procedimentos frequentemente utilizados neste âmbito (e.g., como usar escalas de avaliação, realizar entrevistas, administrar e interpretar testes psicológicos e realizar observações comportamentais). Para além dos motivos legais, especificamente a obrigatoriedade das escolas terem um psicólogo nas suas equipas multidisciplinares (e.g., DL 54/2018), as razões acima expostas mostram que os psicólogos educacionais podem ter um papel importante na avaliação da PHDA e na mediação da informação com outros profissionais de saúde (Brock e Clinton, 2007). Simultaneamente, uma vez que conhecem a perturbação e a forma como se manifesta, podem coordenar as equipas multidisciplinares das escolas no planeamento e desenvolvimento de intervenções psicoeducacionais (Bylund, 2015; Salmon e Kirby, 2009).

Na suspeita de algum caso de PHDA na sala de aula, os professores devem comunicar esta situação ao psicólogo pertencente à equipa multidisciplinar da escola onde leciona. Este profissional deve estar munido de instrumentos que permitam uma avaliação concreta e ser capaz de orientar o professor no preenchimento desta documentação. A EVDPP reúne todas as caraterísticas necessárias para cumprir com este desígnio. Esta avaliação inicial poderá diminuir substancialmente a morosidade do processo avaliativo subsequente e a possibilidade da ocorrência de falsos diagnósticos positivos. 


\section{Referências}

American Psychiatric Association. (2014). Manual de Diagnóstico e Estatística das Perturbações Mentais (5. ${ }^{\mathrm{a}}$ Ed.). Lisboa: Climepsi Editores.

Barkley, R. A., \& Edwards, G. (2008). Entrevista diagnóstica, escalas de avaliação do comportamento e exame médico. In R. A. Barkley et al. (Ed.), Transtorno de Déficit de Atenção/Hiperatividade (3. a ed.) (R. C. Costa, Trad.) (349-400). Porto Alegre: Artmed. (obra original publicada em 2006).

Brislin, R. W. (1970). Back-translation for cross-cultural research. Journal of cross-cultural psychology, 1(3), 185216. https://doi.org/10.1177/135910457000100301

Brislin, R. W. (1976). Comparative research methodology: Cross-cultural studies. International Journal Of Psychology, 11(3), 215-229. https://doi.org/10.1080/00207597608247359

Brock, S. E., \& Clinton, A. (2007). Diagnosis of attention-deficit/hyperactivity disorder (AD/HD) in childhood: A review of the literature. The California School Psychologist, 12(1), 73-91. https://doi.org/10.1007/ BF03340933

Bylund, J. (2015). School Based Assessment of Attention-Deficit/Hyperactivity Disorder. Augusto Guzzo Revista Acadêmica, 1(15), 43-50. Consultado em: http://fics.edu.br/index.php/augusto_guzzo/article/view/263

Conners, C. K. (1969). A teacher rating scale for use in drug studies with children. American journal of Psychiatry, 126(6), 884-888. https://doi.org/10.1176/ajp.126.6.884

Decreto-Lei n ${ }^{\circ}$ 54/2018 de 6 de julho. Diário da República no $129-1^{a}$ Série. Ministério da Educação. Lisboa.

DuPaul, G. J., \& Jimerson, S. R. (2014). Assessing, understanding, and supporting students with ADHD at school: contemporary science, practice, and policy. School psychology quarterly: the official journal of the Division of School Psychology, American Psychological Association, 29(4), 379-384. https://doi.org/10.1037/spq0000104

DuPaul, G. J., Gormley, M. J., \& Laracy, S. D. (2014a). School-based interventions for elementary school students with ADHD. Child and Adolescent Psychiatric Clinics of North America, 23(4), 687-697. https://doi.org/10.1016/j.chc.2014.05.003

DuPaul, G. J., Reid, R., Anastopoulos, A. D., \& Power, T. J. (2014b). Assessing ADHD symptomatic behaviors and functional impairment in school settings: impact of student and teacher characteristics. School Psychology Quarterly: The Official Journal of the Division of School Psychology, American Psychological Association, 29(4), 409-421. https://doi.org/10.1037/spq0000095

Erskine, H., Ferrari, A., Nelson, P., Polanczyk, G., Flaxman, A., Vos, T., \& ... Scott, J. (2013). Research review: Epidemiological modelling of attention-deficit/hyperactivity disorder and conduct disorder for the global burden of disease study 2010. Journal of Child Psychology \& Psychiatry, 54(12), 1263-1274. https://doi.org/10.1111/jcpp.12144

Faraone, S. V., Asherson, P., Banaschewski, T., Biederman, J., Buitelaar, J. k., Ramos-Quiroga, J. A., ... \& Franke, B. (2015). Attention-deficit/hyperactivity disorder. Nature Reviews Disease Primers, 1, 1-23. https://doi.org/ $10.1038 /$ nrdp. 2015.20

Hopkins, W. G. (2002). A scale of magnitudes for effect statistics. A new view of statistics. Acedido em julho 21, 2016, de: http://www.sportsci.org/resource/stats/effectmag.html

Langberg, J. M., Vaughn, A. J., Brinkman, W. B., Froehlich, T., \& Epstein, J. N. (2010). Clinical utility of the Vanderbilt ADHD Rating Scale for ruling out comorbid learning disorders. Pediatrics, 126(5), e1033-e1038. https://doi.org/10.1542/peds.2010-1267

Marôco, J. (2014). Análise estatística com o SPSS Statistics (6. Edição). Pero Pinheiro: ReportNumber.

Oliveira, L., Pereira, M., Medeiros, M., \& Serrano, A. (2016). PHDA: O que há de novo no DSM-5? Revista Portuguesa de Pedagogia, 49(2), 75-94. https://doi.org/10.14195/1647-8614_49-2_4

Rodrigues, A. N. (2007). Escalas revistas de Conners - formas reduzidas para pais e professores. In M. R. Simões, 
C. Machado, M. M. Gonçalves, \& L. S. Almeida (Coord.), Avaliação psicológica: Instrumentos validados para a população portuguesa (Vol. III) (pp.203-227). Coimbra: Edições Quarteto.

Salmon, G., \& Kirby, A. (2009). The role of teachers in the assessment of children suspected of having AD/HD. British Journal of Special Education, 36(3), 147-154. https://doi.org/10.1111/j.1467-8578.2009.00439.x

Shillingford-Butler, M. A., \& Theodore, L. (2013). Students diagnosed with attention deficit hyperactivity disorder: Collaborative strategies for school counselors. Professional School Counseling, 16(4), 235-245. https://doi.org/10.1177/2156759X12016002S05

Schulte-Körne, G. (2016). Mental Health Problems in a School Setting in Children and Adolescents. Deutsches Ärzteblatt International, 113(11), 183-190. https://doi.org/10.3238/arztebl.2016.0183

Weyandt, L. L., \& DuPaul, G. J. (2013). Assessment of ADHD. In College Students with ADHD (37-60). Springer New York. https://doi.org/10.1007/978-1-4614-5345-1_4

Willcutt, E. G. (2012). The prevalence of DSM-IV attention-deficit/hyperactivity disorder: a meta-analytic review. Neurotherapeutics, 9(3), 490-499. https://doi.org/10.1007/s13311-012-0135-8

Wolraich, M. L., Bard, D. E., Neas, B., Doffing, M., \& Beck, L. (2013). The psychometric properties of the Vanderbilt attention-deficit hyperactivity disorder diagnostic teacher rating scale in a community population. Journal of Developmental \& Behavioral Pediatrics, 34(2), 83-93. https://doi.org/10.1097/DBP.0b013e31827d55c3

Wolraich, M. L., Lambert, E. W., Baumgaertel, A., Garcia-Tornel, S., Feurer, I. D., Bickman, L., \& Doffing, M. A. (2003a). Teachers' screening for attention deficit/hyperactivity disorder: Comparing multinational samples on teacher ratings of ADHD. Journal of Abnormal Child Psychology, 31(4), 445-455. https://doi.org/10.1023/ A:1023847719796

Wolraich, M. L., Lambert, E. W., Doffing, M. A., Bickman, L., Simmons, T., \& Worley, K. (2003b). Psychometric properties of the Vanderbilt ADHD diagnostic parent rating scale in a referred population. Journal of Pediatric Psychology, 28(8), 559-568. https://doi.org/10.1093/jpepsy/jsg046

Wolraich, M. L., Feurer, I., Hannah, J., Baumgaertel, A., \& Pinnock, T. (1998). Obtaining systematic teacher reports of disruptive behavior disorders utilizing DSM-IV. Journal of Abnormal Child Psychology, 26(2), 141152. https://doi.org/10.1023/A:1022673906401

\section{Agradecimentos}

Agradece-se ao Dr. José Boavida Fernandes, médico pediatra e responsável da unidade de Hiperatividade do Hospital Pediátrico de Coimbra, pela participação no processo de tradução da EVDPP para Portugués Europeu.

Ao Professor Mark Wolraich, diretor do departamento de Pediatria (Perturbações comportamentais e do desenvolvimento) e diretor do Centro de Estudos da Criança, da Universidade de Ciências da Saúde de Oklahoma, pela autorização da utilização da escala Vanderbilt e revisão da retrodução do instrumento efetuada.

Fecha de recepción: 19 de dciembre de 2018. Fecha de revisión: 3 de junio de 2019. Fecha de aceptación: 7 de junio de 2019. Fecha de publicación: 1 de julio de 2019. 


\section{Apêndices}

\section{Anexo I. Tradução e adaptação dos itens}

\begin{tabular}{|c|c|}
\hline Versão original da escala & Versão final da tradução da escala \\
\hline $\begin{array}{l}\text { 1. Fails to give attention to details or makes careless mistakes } \\
\text { in schoolwork }\end{array}$ & $\begin{array}{l}\text { 1.Tem dificuldade em prestar atenção aos detalhes ou } \\
\text { comete erros por descuido nos trabalhos escolares }\end{array}$ \\
\hline 2. Has difficulty sustaining attention to tasks or activities & $\begin{array}{l}\text { 2.Tem dificuldade em manter a atenção em tarefas ou } \\
\text { atividades }\end{array}$ \\
\hline 3. Does not seem to listen when spoken to directly & 3. Parece não ouvir quando se lhe fala diretamente \\
\hline $\begin{array}{l}\text { 4. Does not follow through on instruction and fails to finish } \\
\text { schoolwork (not due to oppositional behavior or failure to } \\
\text { understand) }\end{array}$ & $\begin{array}{l}\text { 4.Tem dificuldade em seguir as instruções e em terminar os } \\
\text { trabalhos escolares (não por que se recuse ou por não } \\
\text { compreender) }\end{array}$ \\
\hline 5. Has difficulty organizing tasks and activities & 5.Tem dificuldade em organizar tarefas e atividades \\
\hline $\begin{array}{l}\text { 6. Avoids, dislikes, or is reluctant to engage in tasks that } \\
\text { require sustaining mental effort }\end{array}$ & $\begin{array}{l}\text { 6. Evita, não gosta ou mostra-se relutante em envolver-se } \\
\text { em tarefas que exijam um esforço mental sustentado }\end{array}$ \\
\hline $\begin{array}{l}\text { 7. Loses things necessary for tasks or activities (school } \\
\text { assignments, pencils, or books) }\end{array}$ & $\begin{array}{l}\text { 7. Perde material necessário à realização de tarefas ou } \\
\text { atividades (trabalhos da escola, lápis ou livros) }\end{array}$ \\
\hline 8. Is easily distracted by extraneous stimuli & 8. Distrai-se facilmente com estímulos externos \\
\hline 9. Is forgetful in daily activities & 9. É esquecido/a nas atividades quotidianas \\
\hline 10. Fidgets with hands or feet or squirms in seat & $\begin{array}{l}\text { 10. Mexe constantemente as mãos ou pés ou não pára } \\
\text { quieto/a na cadeira }\end{array}$ \\
\hline $\begin{array}{l}\text { 11. Leaves seat in classroom or in other situations in which } \\
\text { remaining seated is expected }\end{array}$ & $\begin{array}{l}\text { 11. Levanta-se na sala de aula ou noutras situações em que } \\
\text { devia permanecer sentado/a }\end{array}$ \\
\hline $\begin{array}{l}\text { 12. Runs about or climbs excessively in situations in which } \\
\text { remaining seated is expected }\end{array}$ & $\begin{array}{l}\text { 12. Corre ou trepa excessivamente em situações em que } \\
\text { devia permanecer sentado/a }\end{array}$ \\
\hline $\begin{array}{l}\text { 13. Has difficulty playing or engaging in leisure activities } \\
\text { quietly }\end{array}$ & $\begin{array}{l}\text { 13. Tem dificuldade em jogar ou em envolver-se } \\
\text { tranquilamente em atividades de lazer }\end{array}$ \\
\hline 14. Is "on the go" or often acts as if "driven by a motor" & $\begin{array}{l}\text { 14. Está sempre em movimento ou age frequentemente } \\
\text { como se fosse "movido/a por um motor" }\end{array}$ \\
\hline 15. T & 15. Fala excessivamente \\
\hline 16. Blurts out answers before questions have been completed & $\begin{array}{l}\text { 16. Responde precipitadamente antes de ouvir as questões } \\
\text { até ao fim }\end{array}$ \\
\hline 17. Has difficulty waiting in line & 17. Tem dificuldade em esperar pela sua vez \\
\hline $\begin{array}{l}\text { 18. Interrupts or intrudes on others (eg, butts into } \\
\text { conversations or games) }\end{array}$ & $\begin{array}{l}\text { 18. Interrompe os outros ou intromete-se (por exemplo, em } \\
\text { conversas ou jogos) }\end{array}$ \\
\hline 19. Loses temper & 19. Perde o controlo \\
\hline $\begin{array}{l}\text { 20. Actively defies or refuses to comply with adults' requests } \\
\text { or rules }\end{array}$ & $\begin{array}{l}\text { 20. Desafia abertamente ou recusa-se a cumprir as ordens } \\
\text { ou regras dos adultos }\end{array}$ \\
\hline 21. Is angry or resentful & 21. Está zangado/a ou ressentido/a \\
\hline 22. Is spiteful and vindictive & 22. É rancoroso/a e vingativo/a \\
\hline 23. Bullies, threatens, or intimidates others & 23. Assusta, ameaça ou intimida os outros \\
\hline 24. Initiates physical fights & 24. Inicia lutas físicas \\
\hline $\begin{array}{l}\text { 25. Lies to obtain goods for favors or to avoid obligations } \\
\text { (ie, "cons" others) }\end{array}$ & $\begin{array}{l}\text { 25. Mente para conseguir bens ou favores ou para evitar } \\
\text { obrigações (isto é, engana os outros) }\end{array}$ \\
\hline 26. Is & amente cruel para as pessoas \\
\hline vial value & 27. $\mathrm{R}$ \\
\hline oroperty & e os bens dos \\
\hline orried & roso/a, ansioso/a ou preocupado/a \\
\hline ly embarr & 30. É tímido/a ou fica envergonhado/a facilmente \\
\hline 31. Is afraid to try new things for fear of making mistakes & $\begin{array}{l}\text { 31. Tem medo de experimentar coisas novas por receio de } \\
\text { cometer erros }\end{array}$ \\
\hline 32. $\mathrm{F}$ & 32. Sente-se inútil ou inferior \\
\hline 33. Blames self for problems, $\mathrm{fe}$ & 33. Culpabiliza-se pelos problemas, sente-se culpado/a \\
\hline $\begin{array}{l}\text { 34. Feels lonely, unwanted, or unloved; complains that " } \\
\text { one loves" him or her }\end{array}$ & $\begin{array}{l}\text { 34. Sente-se sozinho/a, indesejado/a ou pouco amado/a; } \\
\text { reclama que "ninguém gosta dele/a" }\end{array}$ \\
\hline
\end{tabular}


35. Is sad, unhappy, or depressed

\begin{tabular}{l} 
PERFORMANCE \\
\hline Academic Performance \\
1. Reading \\
2. Mathematics \\
3. Written expression
\end{tabular}

Classroom Behavioral Performance

1. Relationships with peers

2. Following directions/rules

3. Disrupting class

4. Assignment completion

5. Organizational skills
35. Está triste, infeliz ou deprimido/a

DESEMPENHO

Desempenho académico

1. Leitura

2. Matemática

3. Expressão escrita

Desempenho comportamental em sala de aula

1. Relacionamento com os colegas

2. Cumprimento de ordens/regras

3. Perturbação das aulas

4. Conclusão de tarefas

5. Capacidade de organização

\section{Anexo II. Retrotradução dos itens}

\begin{tabular}{|c|c|}
\hline Versão original da escala & Versão final da retrotradução da escala \\
\hline $\begin{array}{l}\text { 1. Fails to give attention to details or makes careless mistakes } \\
\text { in schoolwork }\end{array}$ & $\begin{array}{l}\text { 1. Has difficulty paying attention to details or makes } \\
\text { careless mistakes on schoolwork }\end{array}$ \\
\hline 2. Has difficulty sustaining attention to tasks or activities & 2. Has difficulty maintaining focus on tasks or activities \\
\hline 3. Does not seem to listen when spoken to directly & 3. Seems to not hear when being spoken to directly \\
\hline $\begin{array}{l}\text { 4. Does not follow through on instruction and fails to finish } \\
\text { schoolwork (not due to oppositional behavior or failure to } \\
\text { understand) }\end{array}$ & $\begin{array}{l}\text { 4. Has difficulty following directions and does not finish } \\
\text { schoolwork (not because of refusal or lack of } \\
\text { understanding) }\end{array}$ \\
\hline 5. Has difficulty organizing tasks and activities & 5. Has difficulty organizing tasks and activities \\
\hline $\begin{array}{l}\text { 6. Avoids, dislikes, or is reluctant to engage in tasks that } \\
\text { require sustaining mental effort }\end{array}$ & $\begin{array}{l}\text { 6. Avoids, dislikes or is reluctant to engage in tasks that } \\
\text { require sustained mental effort }\end{array}$ \\
\hline $\begin{array}{l}\text { 7. Loses things necessary for tasks or activities (school } \\
\text { assignments, pencils, or books) }\end{array}$ & $\begin{array}{l}\text { 7. Loses materials needed to perform tasks or activities } \\
\text { (schoolwork, pencils or books) }\end{array}$ \\
\hline distracted by extraneous stimuli & 8. Easily distracted by external stimuli \\
\hline ful in daily activities & 9. Is forgetful in daily activities \\
\hline 10. Fidgets with hands or feet or squirms in seat & $\begin{array}{l}\text { 10. Constantly moving hands or feet or does not sit quietly } \\
\text { in the chair }\end{array}$ \\
\hline $\begin{array}{l}\text { 11. Leaves seat in classroom or in other situations in which } \\
\text { remaining seated is expected }\end{array}$ & $\begin{array}{l}\text { 11. Gets up in the classroom or in other situations where } \\
\text { he/she should remain seated }\end{array}$ \\
\hline $\begin{array}{l}\text { 12. Runs about or climbs excessively in situations in which } \\
\text { remaining seated is expected }\end{array}$ & $\begin{array}{l}\text { 12. Runs or climbs excessively in situations where he/she } \\
\text { should remain seated }\end{array}$ \\
\hline $\begin{array}{l}\text { 13. Has difficulty playing or engaging in leisure activities } \\
\text { quietly }\end{array}$ & $\begin{array}{l}\text { 13. Has difficulty calmly playing or engaging in leisure } \\
\text { activities }\end{array}$ \\
\hline 14. Is "on the go" or often acts as if "driven by a motor" & $\begin{array}{l}\text { 14. Is always moving or often acts as though he/she is } \\
\text { "driven by a motor" }\end{array}$ \\
\hline 15. 7 & 15. $\mathrm{T}$ \\
\hline e questions have been completed & ne entire question \\
\hline 17. Has difficulty waiting in line & ficulty waiting for his/her turn \\
\hline $\begin{array}{l}\text { 18. Interrupts or intrudes on others (eg, butts into } \\
\text { conversations or games) }\end{array}$ & $\begin{array}{l}\text { 18. Interrupts others or interferes (for example, in } \\
\text { conversations or games) }\end{array}$ \\
\hline 19. Loses temper & 19. Loses control \\
\hline $\begin{array}{l}\text { 20. Actively defies or refuses to comply with adults' requests } \\
\text { or rules }\end{array}$ & $\begin{array}{l}\text { 20. Openly challenges or refuses to comply with adults' } \\
\text { orders or rules }\end{array}$ \\
\hline 21. Is angry & 21. Is \\
\hline 22. I & 22 \\
\hline timidates others & or intimida \\
\hline 24. I & hysical fights \\
\hline $\begin{array}{l}\text { 25. Lies to obtain goods for favors or to avoid obligations } \\
\text { (ie, "cons" others) }\end{array}$ & $\begin{array}{l}\text { 25. Lies to get goods or favors or to avoid obligations (i.e. } \\
\text { deceiving others) }\end{array}$ \\
\hline 26. Is physically cruel to people & 26. Is physically cruel to people \\
\hline
\end{tabular}


27. Has stolen items of nontrivial value

28. Deliberately destroys others' property

29. Is fearful, anxious, or worried

30. Is self-conscious or easily embarrassed

31. Is afraid to try new things for fear of making mistakes

32. Feels worthless or inferior

33. Blames self for problems, feels guilty

34. Feels lonely, unwanted, or unloved; complains that "no one loves" him or her

35. Is sad, unhappy, or depressed

\section{PERFORMANCE}

Academic Performance

1. Reading

2. Mathematics

3. Written expression

Classroom Behavioral Performance

1. Relationships with peers

2. Following directions/rules

3. Disrupting class

4. Assignment completion

5. Organizational skills
27. Steals objects of great value

28. Deliberately destroys others' property

29. Is fearful, anxious or worried

30. Is shy and gets embarrassed easily

31. Is afraid of trying new things for fear of making mistakes

32. Feels useless or inferior

33. Blames himself/herself for problems, feels guilty

34. Feels alone, unwanted or unloved; complains that "nobody likes him/her"

35. Is sad, unhappy or depressed

\section{PERFORMANCE}

Academic Performance

1. Reading

2. Mathematics

3. Writing skills

Behavioral performance in the classroom

1. Relationships with peers

2. Compliance with orders/rules

3. Classroom disruption

4. Completion of tasks

5. Organizational skills 